\title{
A direct proof of dimerization in a family of $S U(n)$-invariant quantum spin chains
}

\author{
Bruno Nachtergaele ${ }^{1}$ - Daniel Ueltschi ${ }^{2}$
}

Received: 23 January 2017 / Revised: 23 March 2017 / Accepted: 29 March 2017 /

Published online: 9 May 2017

(C) The Author(s) 2017. This article is an open access publication

\begin{abstract}
We study the family of spin- $S$ quantum spin chains with a nearest neighbor interaction given by the negative of the singlet projection operator. Using a random loop representation of the partition function in the limit of zero temperature and standard techniques of classical statistical mechanics, we prove dimerization for all sufficiently large values of $S$.
\end{abstract}

Keywords Quantum spin chain · Dimerization $\cdot S U(n)$-invariant chains

Mathematics Subject Classification $82 \mathrm{~B} 10 \cdot 82 \mathrm{~B} 20 \cdot 82 \mathrm{~B} 26$

\section{Introduction}

Dimerization is the most common type and the most common mechanism of spontaneous lattice translation symmetry breaking in the ground state of quantum spin systems. It is ubiquitous in one dimension due to the so-called (spin-) Peierls instability [9]. In two and more dimensions it gives rise to columnar phases and other patterns of lattice symmetry breaking $[12,13,23,24]$. In the ground states of quantum spin chains with isotropic antiferromagnetic interactions, long-range antiferromagnetic order, and the accompanying spontaneous breaking of the continuous rotation symmetry, is prevented by quantum fluctuations. Short-range antiferromagnetic cor-

Daniel Ueltschi

daniel@ueltschi.org

Bruno Nachtergaele

bxn@math.ucdavis.edu

1 Department of Mathematics, University of California, One Shields Ave, Davis, CA 95616, USA

2 Department of Mathematics, University of Warwick, Coventry CV4 7AL, UK 
relations, however, occur in several manifestations, some of which involve discrete symmetry breaking. Due to a result by Affleck and Lieb [3], half-integer spin chains with a rotation symmetry and a unique ground state have a gapless excitation spectrum above the ground state. The opening up of a non-vanishing (in the thermodynamic limit) gap above the ground state requires that the ground state is degenerate, and for a class of reflection positive antiferromagnetic chains it is known that the nature of this degeneracy is dimerization, i.e., breaking of the translation invariance from $\mathbb{Z}$ to $2 \mathbb{Z}[4,21]$.

In this paper we study a family of spin- $S$ chains with an isotropic nearest neighbor Hamiltonian for a chain of $L$ spins given by

$$
H(S, L)=-\sum_{x=1}^{L-1} P_{x, x+1}^{(0)},
$$

where $P_{x, x+1}^{(0)}$ denotes the orthogonal projection onto the singlet state of the two spins at sites $x$ and $x+1$. Since $P_{x, x+1}^{(0)}$ commutes with the tensor product of the fundamental and the anti-fundamental representation of $S U(2 S+1)$, this model has an $S U(2 S+1)$ symmetry. For $S=1 / 2$ this Hamiltonian is, up to a constant, the standard spin-1/2 antiferromagnetic Heisenberg chain and its Bethe ansatz gives a unique, translation-invariant ground state with power law correlations and a gapless excitation spectrum [17]. For $S \geq 1$ the ground state is expected to be dimerized and twofold degenerate, and with a positive spectral gap. This expectation is supported by relationships between the spin chain Hamiltonian $H(S, L)$ and other spin Hamiltonians that are diagonalizable using the Bethe ansatz or other exact solution methods $[1,5,16]$; e.g., the $S=1$ model can be related to spin- $1 / 2 X X Z$ chain with anisotropy $\Delta=-3 / 2$ and to the transfer matrix of the standard 9-state Potts model on a square lattice. These relationships stem from the observation that $H(S, L)$ and the related spin chain Hamiltonian or transfer matrix are both representatives of an element $H_{N}$ in the abstract Temperley-Lieb algebra $T L_{N}(x)$, with $x=2 S+1$. This implies that the spectrum of each of these operators is a subset of the algebraic spectrum of $H_{N}$, i.e., the complex numbers $\lambda$ for which $H_{N}-\lambda \mathbb{l}$ fails to have an inverse in $T L_{N}(x)$. Many algebras have only one irreducible representation, in which case the spectrum is the same in any representation with only the multiplicity remaining to be determined. The number of irreducible representations of the Temperley-Lieb algebra $T L_{N}(x)$, however, grows as $\sim N^{2}$, and two representations could therefore, in principle, give entirely different spectra to the element $H_{N}$. Nevertheless, exact calculations on small chains and numerical calculations indicate that the spectra coincide up to multiplicity. More recent results on the algebraic Temperley-Lieb chains may point to an explanation of this "universality" of the spectrum. See [22] and the references therein for a discussion of this phenomenon.

The relationship between the spin- $S$ chain with Hamiltonian (1.1) with $S \geq 1$ and the two-dimensional $q$-state Potts model with $q=(2 S+1)^{2}$ at the self-dual point extends in a non-trivial way to the states: The two dimerized ground states correspond to the coexisting ordered and disordered phases of the $q$-state Potts model on the square 
lattice at its critical point. This coexistence (a first-order phase transition) has been found by Baxter [6] for $q \geq 5$ and has been rigorously established for all sufficiently large values of $q$ [18]. Even if one accepts the identity of the spectra of the related Hamiltonians, relating order parameters or, in general, the states of these models is a subtle issue. In [4] it was shown that the dimerization order parameter for the spin chain and the order parameter of a suitable two-dimensional ferromagnetic Potts model are bounded by a multiple of each other. This implies that one of them vanishes if and only if the other does. The Potts model for which this relationship is proved can be regarded as a strongly anisotropic limit (with respect to the two lattice directions), in which one of the lattice directions tends to the continuum. A similar result likely also holds for the standard Potts model on the square lattice, but this has not been worked out in the literature.

Due to the subtleties of the relationships between the different models discussed above, a complete proof of dimerization has been lacking. In view of the non-trivial nature of rigorous studies of the critical Potts model itself, it seemed worthwhile to look for a direct proof of the dimerization, bypassing establishing more details of the relationship between the various models. In this article we provide the first complete proof of the existence of dimerized ground states for this class of models at sufficiently large values of $S$. Moreover, by not relying on the Temperley-Lieb algebra and a relation to the Potts models, we open the possibility to study perturbations of the model away from the self-dual point in the phase diagram. This is important in the context of the current interest in stable gapped ground state phases of quantum lattice systems, which we briefly discuss in Sect. 5.

Our approach is based on a random loop representation for the partition function of the spin models (1.1) given in [4], which has been applied in recent years for a number of other rigorous results for quantum spin models [7, 8, 19]. For a review and extensions of the random loop representation for quantum spin models, see [25]. We give a precise statement of our results in the next section. A detailed description of the random loop representation and its properties is given in Sect. 3. In Sect. 4 we introduce a suitable notion of contours and the Peierls argument proof of our main result. We conclude with a short discussion.

\section{Setting and results}

For $\ell \in \mathbb{N}$, consider a chain of even length $2 \ell$ consisting of the set of vertices $\{-\ell+$ $1,-\ell+2, \ldots, \ell\}$ with edges between nearest neighbors. For $S \in \frac{1}{2} \mathbb{N}$, the Hilbert space of a spin- $S$ chain is then

$$
\mathcal{H}_{\ell}=\otimes_{x=-\ell+1}^{\ell} \mathbb{C}^{2 S+1}
$$

The interaction is nearest neighbor and given by the orthogonal projection $P_{x, x+1}^{(0)}$ onto the spin singlet at the sites $x$ and $x+1$. In terms of the standard tensor product basis of $\mathbb{C}^{2 S+1} \otimes \mathbb{C}^{2 S+1}$ constructed with the eigenvectors on the third component of the 
spin, this operator is given by

$$
P_{x, y}^{(0)}=\frac{1}{2 S+1} \sum_{a, b=-S}^{S}(-1)^{a-b}|a,-a\rangle\langle b,-b| .
$$

We also write $P_{x, x+1}^{(0)}$ for the operator on $\mathcal{H}_{\ell}$, obtained by tensoring it with the identity on the Hilbert spaces corresponding to sites other than $x$ and $x+1$. The Hamiltonian of the models studied in this paper is

$$
H_{\ell}=-\sum_{x=-\ell+1}^{\ell-1} P_{x, x+1}^{(0)}
$$

The interaction can be written in terms of the usual spin operators $S_{x}^{i}, i=1,2,3$, which satisfy

$$
\left[S_{x}^{1}, S_{y}^{2}\right]=\delta_{x, y} \mathrm{i} S_{x}^{3},
$$

and further relations obtained by cyclic permutations of the indices $1,2,3$, and also satisfy

$$
\left(S_{x}^{1}\right)^{2}+\left(S_{x}^{2}\right)^{2}+\left(S_{x}^{3}\right)^{2}=S(S+1) .
$$

For instance, for $S=1 / 2,1$, and $3 / 2$, one has

$$
\begin{array}{ll}
S=\frac{1}{2}: & P_{x, y}^{(0)}=-\vec{S}_{x} \cdot \vec{S}_{y}+\frac{1}{4} \\
S=1: & P_{x, y}^{(0)}=\frac{1}{3}\left(\vec{S}_{x} \cdot \vec{S}_{y}\right)^{2}-\frac{1}{3} \\
S=\frac{3}{2}: & P_{x, y}^{(0)}=-\frac{1}{18}\left(\vec{S}_{x} \cdot \vec{S}_{y}\right)^{3}-\frac{5}{72}\left(\vec{S}_{x} \cdot \vec{S}_{y}\right)^{2}+\frac{31}{96} \vec{S}_{x} \cdot \vec{S}_{y}+\frac{33}{128} .
\end{array}
$$

The $S=\frac{1}{2}$ model is the usual Heisenberg antiferromagnet, and it is not expected to display long-range order in its one-dimensional ground state. Dimerization is expected for $S \geq 1$, and in this paper we prove it for $S \geq 40$. More specifically, we derive a lower bound for a dimerization parameter order in the zero-temperature limit of Gibbs states on even chains, uniformly in the length of the chain. To state our result, let $\langle\cdot\rangle_{\beta, \ell}$ denote the Gibbs state,

$$
\langle a\rangle_{\beta, \ell}=\frac{1}{\operatorname{Tr} \mathrm{e}^{-\beta H_{\ell}}} \operatorname{Tr} a \mathrm{e}^{-\beta H_{\ell}},
$$

and let $\langle\cdot\rangle_{\infty, \ell}=\lim _{\beta \rightarrow \infty}\langle\cdot\rangle_{\beta, \ell}$ denote the expectation in the ground state. Our main result is the following theorem.

Theorem 2.1 For $S \geq 40$, there exists $c(S)>0$ such that

$$
\left\langle P_{x, x+1}^{(0)}\right\rangle_{\infty, \ell}-\left\langle P_{x-1, x}^{(0)}\right\rangle_{\infty, \ell}>c(S)
$$

for all $x \in\{-\ell+3,-\ell+5, \ldots, \ell-1\}$, uniformly in $\ell \in \mathbb{N}$. 


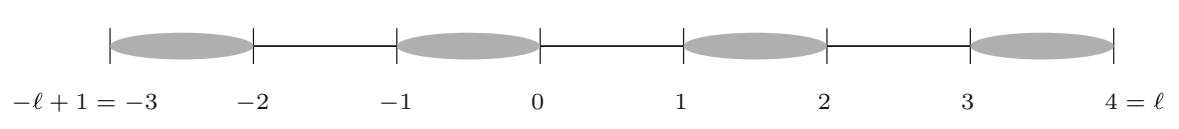

Fig. 1 Dimerization in a chain of length $\ell=4$

The theorem states that, for any $x \in\{-\ell+3,-\ell+5, \ldots, \ell-1\}$, the probability that the bond $\{x, x+1\}$ is in the singlet state (dimerized) exceeds the probability that $\{x-1, x\}$ is in the singlet state by a positive amount, uniformly in $\ell$. See Fig. 1 for an illustration. Thus, in the limit $\ell \rightarrow \infty$ along even values, one gets a ground state where $\{-1,0\}$ is more likely to be dimerized than $\{0,1\}$. In the limit $\ell \rightarrow \infty$ along odd values, the converse is true. This establishes the existence of two distinct, nontranslation-invariant ground states. By an averaging procedure, one sees that there are two periodic ground states of period 2 . We conjecture that these are the only ground states of the infinite chain. The proof of Theorem 2.1 is found at the end of Sect. 4.

An open question is whether dimerization occurs in higher dimensions. The presence of Néel order has been proved for $S=\frac{1}{2}$ and $d \geq 3$ [11], and for any $S \geq 1$ provided the dimension is large enough (depending on $S$ ) [25]. This leaves open the possibility that for fixed $d$, such as $d=2$, dimerization occurs when $S$ is large enough.

Our proof of dimerization is based on the random loop representation of [4]. We introduce excitation contours in a background of dimerized short loops, a setting that allows to use a Peierls argument. It is presented in Sect. 4. More precisely, Theorem 2.1 is an immediate consequence of Proposition 4.4 combined with Proposition 3.1, Lemma 3.2, and Proposition 3.3.

The ground state is no longer translation invariant, but it still has spin rotation invariance, as shown in the following theorem. In particular, there is no Néel order. In fact, this theorem implies that in the two periodic ground states all correlations (not just spin-spin correlations) decay exponentially and therefore are extremal periodic ground states. This supports our conjecture that they are the only ground states of the infinite chain.

Theorem 2.2 For $S \geq 8$, there exist $C, \eta>0$ such that

$$
\left|\left\langle S_{x}^{i} S_{y}^{j}\right\rangle_{\infty, \ell}\right| \leq C \mathrm{e}^{-|x-y| / \eta},
$$

for all $i, j=1,2,3$, all $x, y \in\{-\ell+1, \ldots, \ell\}$, and all $\ell \in \mathbb{N}$.

The proof of this theorem is found at the end of Sect. 4; it is based on the same properties of the random loop representation that we prove for Theorem 2.1. Correlations between $x$ and $y$ can be expressed in terms of events in which loops (contours) connect $x$ to $y$, and which require that these points are surrounded by contours of size larger than $|x-y|$. The probability of these contours decays exponentially fast with respect to their size. Because all correlation functions can be expressed in terms of loop connectivity (see [4]), it follows that all correlations decay exponentially. Notice that Theorem 2.2 holds for smaller $S$ than Theorem 2.1; the reason is that it only involves large loops. 
Theorem 2.2 also implies that the translation symmetry is spontaneously broken in the ground state for all $S \geq 8$. This follows from [4, Theorem 6.1]. For $S \geq 40$, Theorem 2.1 gives a quantitative estimate of this non-translation invariance in terms of the probability that two nearest neighbor spins form a dimer.

\section{Loop representation and contours}

We now give a description of the random loop representation of the Gibbs states defined in (2.7). It is convenient to restrict to $\beta \in \mathbb{N}$.

\subsection{Loops}

Let $E_{\ell}=\{\{x, x+1\}:-\ell+1 \leq x \leq \ell-1\}$ denote the set of edges. We also introduce the set $E_{\ell}^{1}$ of edges that are expected to preferentially host dimers (those that are shaded in Fig. 1)

$$
E_{\ell}^{1}=\{\{x, x+1\}: x \in\{-\ell+1,-\ell+3, \ldots, \ell-1\}\},
$$

and $E_{\ell}^{2}=E_{\ell} \backslash E_{\ell}^{1}$. The length of the chain is chosen to be even, and this, it turns out, makes it more likely that we have dimers at both edges of the chain. This is the mechanism that will select one of the two ground states.

In contrast to previous applications of the random loop representation [4,25], it will be convenient for us here to use a discrete version of the loop representation, defined as follows. Let $n \in \mathbb{N}$, and consider the set $T_{\beta, n}$ of discrete times, $T_{\beta, n}=\frac{1}{n} \mathbb{Z} \cap[-\beta, \beta]$. A configuration $\omega$ is a subset of $E_{\ell} \times T_{\beta, n}$; we say that a "double bar" is present at $\{x, x+1\} \times t \in E_{\ell} \times T_{\beta, n}$ whenever $\{x, x+1\} \times t \in \omega$; there are no double bars at $\{x, x+1\} \times t$ otherwise. We let $\Omega_{\ell, n}$ denote the set of configurations where no more than one double bar occurs at any given time; it is also useful to exclude double bars at time 0 .

To a configuration $\omega \in \Omega_{\ell, n}$ corresponds a set of loops, as illustrated in Fig. 2. A loop $\gamma$ is a closed trajectory

$$
\begin{aligned}
{[0, L]_{\mathrm{per}} } & \rightarrow\{-\ell+1, \ldots, \ell\} \times[-\beta, \beta]_{\mathrm{per}} \\
t & \mapsto \gamma(t)=(x(t), T(t))
\end{aligned}
$$

such that $x(t)$ is piecewise constant and $T^{\prime}(t)= \pm 1$. We let $L(\gamma)$ denote the length of $\gamma$, that is, the smallest $L>0$ in the above equation. A jump occurs at $t \in[0, L(\gamma)]$ provided $\{x(t-), x(t+)\} \times T(t)$ contains a double bar, and that $T^{\prime}(t-)=-T^{\prime}(t+)$. We identify loops with identical support. Let $\mathcal{L}(\omega)$ denote the number of loops of the configuration $\omega$. We always have $1 \leq \mathcal{L}(\omega) \leq 2 \ell+|\omega|$ and

$$
\sum_{j=1}^{\mathcal{L}(\omega)} L\left(\gamma_{j}\right)=2 \beta \ell .
$$




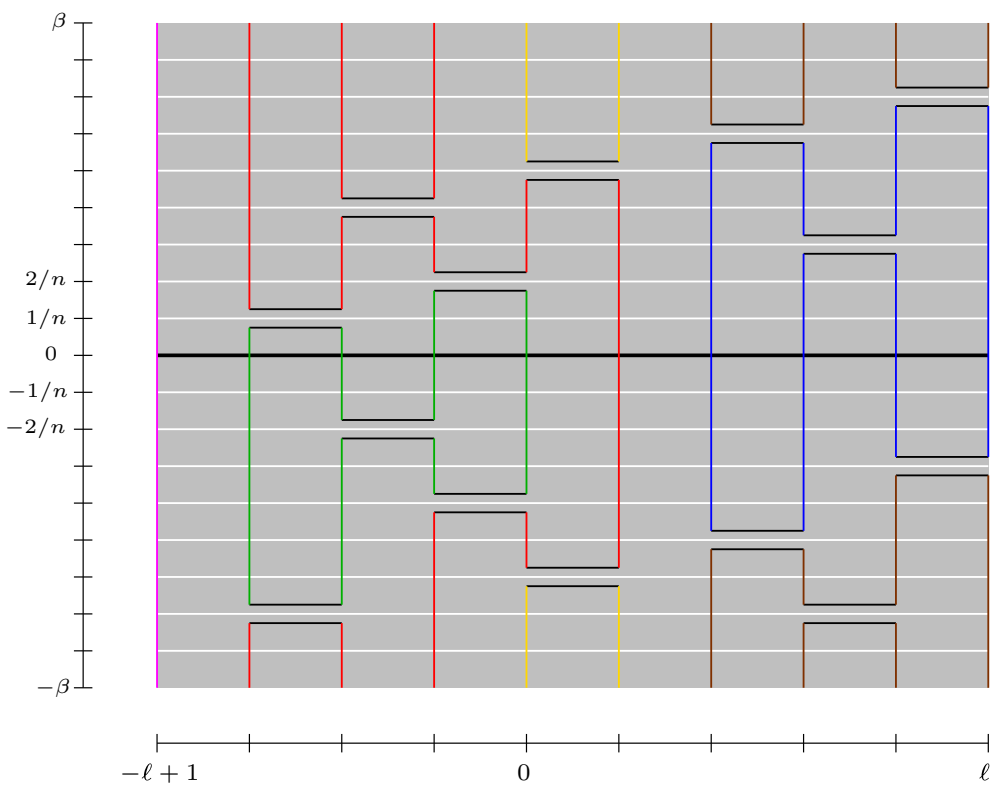

Fig. 2 Loop representation of the $S U(2 S+1)$-invariant quantum spin chains

The relevant probability measure on $\Omega_{\ell, n}$ involves the number of loops and is given by

$$
\mu_{\beta, \ell, n}(\omega)=\frac{1}{Z_{n}(\beta, \ell)}\left(\frac{1}{n}\right)^{|\omega|}(2 S+1)^{\mathcal{L}(\omega)-|\omega|},
$$

with

$$
Z_{n}(\beta, \ell)=\sum_{\omega \in \Omega_{\ell, n}}\left(\frac{1}{n}\right)^{|\omega|}(2 S+1)^{\mathcal{L}(\omega)-|\omega|} .
$$

In the following, we use the notation $\mathbb{P}_{\beta, \ell, n}$ and $\mathbb{E}_{\beta, \ell, n}$ for the probability and expectation with respect to the measure $\mu_{\beta, \ell, n}$. The event $x \leftrightarrow y$ (resp. $x \leftrightarrow y$ ) represents the set of all $\omega \in \Omega_{\ell, n}$ where $x \times 0$ and $y \times 0$ belong to the same loop (resp. belong to distinct loops).

Proposition 3.1 (a) $\operatorname{Tr} \mathrm{e}^{-2 \beta H_{\ell}}=\lim _{n \rightarrow \infty} Z_{n}(\beta, \ell)$.

(b) $\left\langle P_{x, x+1}^{(0)}\right\rangle_{2 \beta, \ell}=\frac{1}{(2 S+1)^{2}}+\left(1-\frac{1}{(2 S+1)^{2}}\right) \lim _{n \rightarrow \infty} \mathbb{P}_{\beta, \ell, n}(x \leftrightarrow x+1)$.

Proof We only prove (b), since the proof of (a) is similar and it can be found in [4]. Using the Trotter formula, we have 


$$
\begin{aligned}
\operatorname{Tr} \mathrm{e}^{-\beta H_{\ell}} P_{x, x+1}^{(0)} \mathrm{e}^{-\beta H_{\ell}}= & \lim _{n \rightarrow \infty} \operatorname{Tr} \prod_{\{y, y+1\} \times t \in E_{\ell} \times T_{\beta, n}}\left[1+\frac{1}{n} P_{y, y+1}^{(0)}\right] \\
& P_{x, x+1}^{(0)} \prod_{\{y, y+1\} \times t \in E_{\ell} \times T_{\beta, n}}\left[1+\frac{1}{n} P_{y, y+1}^{(0)}\right] \\
= & \lim _{n \rightarrow \infty} \sum_{\omega \in \Omega_{\ell, n}}\left(\frac{1}{n}\right)^{|\omega|} \operatorname{Tr} \prod_{\{y, y+1\} \times t \in \bar{\omega}} P_{y, y+1}^{(0)} .
\end{aligned}
$$

Here, the configuration $\bar{\omega}$ is equal to the configuration $\omega$ with an extra double bar at $\{x, x+1\} \times 0$, and the last product is ordered by increasing times $t \in T_{\beta, n}$. It is not hard to verify that

$$
\operatorname{Tr} \prod_{\{y, y+1\} \times t \in \bar{\omega}} P_{y, y+1}^{(0)}=(2 S+1)^{\mathcal{L}(\bar{\omega})-|\bar{\omega}|} .
$$

Next, observe that

$$
\mathcal{L}(\bar{\omega})= \begin{cases}\mathcal{L}(\omega)+1 & \text { if } x \leftrightarrow x+1 ; \\ \mathcal{L}(\omega)-1 & \text { if } x \leftrightarrow x+1 .\end{cases}
$$

As a consequence, we have

$$
\begin{aligned}
\operatorname{Tr} \mathrm{e}^{-\beta H_{\ell}} P_{x, x+1}^{(0)} \mathrm{e}^{-\beta H_{\ell}} & =\lim _{n \rightarrow \infty} \sum_{\omega \in \Omega_{\ell, n}}\left(\frac{1}{n}\right)^{|\omega|}(2 S+1)^{\mathcal{L}(\omega)-|\omega|-1} \\
& {\left[(2 S+1) 1_{x \leftrightarrow x+1}(\omega)+\frac{1}{2 S+1} 1_{x \leftrightarrow x+1}(\omega)\right] } \\
= & \lim _{n \rightarrow \infty} Z_{n}(\beta, \ell)\left[\mathbb{P}_{\beta, \ell, n}(x \leftrightarrow x+1)+\frac{1}{(2 S+1)^{2}} \mathbb{P}_{\beta, \ell, n}(x \leftrightarrow x+1)\right],
\end{aligned}
$$

and the claim (b) of the proposition follows.

The measure $\mu_{\beta, \ell, n}$ is biased toward configurations with many loops. The optimal way to stack many loops is to have only double bars on edges $\{x, x+1\} \in E_{\ell}^{1}$, see Fig. 3. If $\omega$ has this form, we have

$$
1_{x \leftrightarrow x+1}(\omega)= \begin{cases}1 & \text { if }\{x, x+1\} \in E_{\ell}^{1}, \\ 0 & \text { if }\{x, x+1\} \in E_{\ell}^{2} .\end{cases}
$$

If these were typical configurations, we would obtain $\mathbb{P}_{\beta, \ell, n}(x \leftrightarrow x+1)=1>$ $0=\mathbb{P}_{\beta, \ell, n}(x \leftrightarrow x+1)$ for all $x \in\{-\ell+1,-\ell+3, \ldots, \ell-1\}$, and Theorem 2.1 follows from Proposition 3.1. Actual typical configurations are naturally more complicated but, for large $S$, not very much, as they possess this structure up to sparse "excitations." This is shown below. We put excitations in quotes here because they are present with a nonzero density in the ground states and can be regarded as representing the quantum fluctuations at zero temperature. 


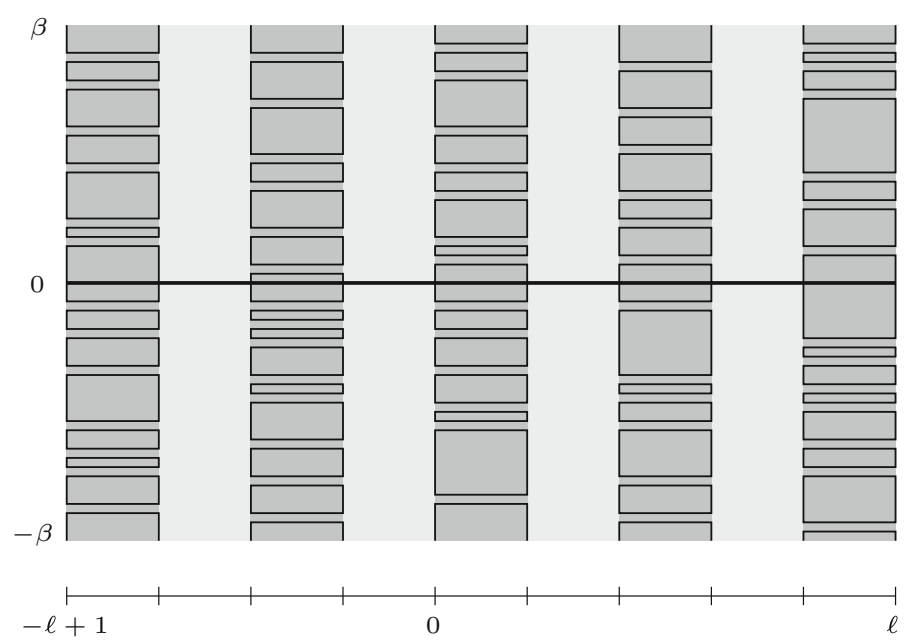

Fig. 3 Illustration for "optimal" configurations that allow for large numbers of loops

\subsection{Simplified set of configurations without winding loops}

As is usual, we will derive our estimates for finite systems, which in our case means finite chains at finite inverse temperature $\beta$. The estimates will then carry over to the limit of infinite $\beta$ and the infinite chain. In the limit $\beta \rightarrow \infty$, the so-called winding loops will have vanishing probability. These winding loops are a complication for the Peierls-type argument we want to develop. Therefore, it will be helpful to work with a restricted set of configurations in which almost all winding loops have been eliminated. To do this we need to show that the probability of the configurations we ignore indeed vanishes in the limit $\beta \rightarrow \infty$. This is the purpose of the next lemma.

Given an integer $\alpha<\beta$, let $\Omega_{\ell, n}^{\alpha}$ denote the set of configurations where intervals $\{x, x+1\} \times[\alpha, \alpha+1]$ contain at least one double bar if $\{x, x+1\} \in E_{\ell}^{1}$, and none if $\{x, x+1\} \in E_{\ell}^{2}$. These configurations possess a convenient, spontaneous boundary condition in the time direction (this is depicted in Fig. 5). Almost all configurations have this property for some $\alpha \in \mathbb{N}$ :

Lemma 3.2 We have

$$
\lim _{\beta \rightarrow \infty} \mathbb{P}_{\beta, \ell, n}\left(\cup_{\alpha=1}^{\beta} \Omega_{\ell, n}^{\alpha}\right)=1
$$

The limit $\beta \rightarrow \infty$ converges uniformly in $n$.

Proof Let $A_{\alpha}=\cup_{\alpha^{\prime}<\alpha} \Omega_{\ell, n}^{\alpha^{\prime}}$. We have $A_{\alpha+1} \supset A_{\alpha}$ for all $\alpha \in \mathbb{N}$, and one checks below that

$$
\mathbb{P}_{\beta, \ell, n}\left(A_{\alpha+1} \mid A_{\alpha}^{\mathrm{c}}\right)>c,
$$

with $c>0$ independent of $\alpha, \beta, n$ (it depends on $S, \ell$, though). Then

$$
\mathbb{P}_{\beta, \ell, n}\left(A_{\beta}^{\mathrm{c}}\right)=\mathbb{P}_{\beta, \ell, n}\left(A_{\beta}^{\mathrm{c}} \mid A_{\beta-1}^{\mathrm{c}}\right) \mathbb{P}_{\beta, \ell, n}\left(A_{\beta-1}^{\mathrm{c}}\right) \leq(1-c) \mathbb{P}_{\beta, \ell, n}\left(A_{\beta-1}^{\mathrm{c}}\right)
$$


Iterating, we find that $\mathbb{P}_{\beta, \ell, n}\left(A_{\beta}^{\mathrm{c}}\right)$ is less than $(1-c)^{\beta}$, which implies the lemma.

In order to check (3.11), let $\hat{A}_{\alpha} \subset A_{\alpha}^{\mathrm{c}}$ be the set of configurations such that no double bars occur at times in $[\alpha, \alpha+1] \cap T_{\beta, n}$. A configuration $\omega \in A_{\alpha}^{\mathrm{c}}$ can be decomposed as $\omega=\hat{\omega} \cup \omega^{\prime}$, where $\hat{\omega} \in \hat{A}_{\alpha}$ and $\omega^{\prime}$ contain only double bars at times in $[\alpha, \alpha+1] \cap T_{\beta, n}$. We have

$$
\mathcal{L}(\hat{\omega})-\left|\omega^{\prime}\right| \leq \mathcal{L}(\omega) \leq \mathcal{L}(\hat{\omega})+\left|\omega^{\prime}\right|
$$

so that

$$
\mu_{\beta, \ell, n}(\hat{\omega})\left(\frac{1}{n}\right)^{\left|\omega^{\prime}\right|}(2 S+1)^{-2\left|\omega^{\prime}\right|} \leq \mu_{\beta, \ell, n}(\omega) \leq \mu_{\beta, \ell, n}(\hat{\omega})\left(\frac{1}{n}\right)^{\left|\omega^{\prime}\right|}
$$

Then

$$
\begin{aligned}
\mathbb{P}_{\beta, \ell, n}\left(A_{\alpha+1} \mid A_{\alpha}\right) & =\frac{\mathbb{P}_{\beta, \ell, n}\left(\Omega_{\ell, n}^{\alpha} \cap A_{\alpha}^{\mathrm{c}}\right)}{\mathbb{P}_{\beta, \ell, n}\left(A_{\alpha}^{\mathrm{c}}\right)}=\frac{\sum_{\hat{\omega} \in \hat{A}_{\alpha}} \sum_{\omega^{\prime}: \Omega_{\ell, n}^{\alpha}} \mu_{\beta, \ell, n}\left(\hat{\omega} \cup \omega^{\prime}\right)}{\sum_{\hat{\omega} \in \hat{A}_{\alpha}} \sum_{\omega^{\prime}} \mu_{\beta, \ell, n}\left(\hat{\omega} \cup \omega^{\prime}\right)} \\
& \geq \frac{\left(1+\frac{1}{n}(2 S+1)^{-2}\right)^{\ell n}}{\left(1+\frac{1}{n}\right)^{(2 \ell+1) n}} .
\end{aligned}
$$

The last term is indeed bounded away from 0 , uniformly in $\alpha, \beta, n$.

Loops visiting only one or two sites are called short loops. The loops shown in Fig. 3 are all short loops. We say that a loop is long if it is not short. Since the loops of configurations of $\Omega_{\ell, n}^{\alpha}$ do not wind, they have an interior in the sense of Jordan curves; we actually call "interior" its intersection with $\{-\ell+1, \ldots, \ell\} \times T_{\beta, n}$. For $\omega \in \Omega_{\ell, n}^{\alpha}$, we introduce the event $E_{x}^{\circlearrowleft}$ where $(x, 0)$ belongs to a long loop, or to the interior of a long loop. See Eq. (4.1) for an equivalent definition that involves contours, to be defined below.

Proposition 3.3 If $x \in\{-\ell+3,-\ell+5, \ldots, \ell-1\}$, we have

$$
\mathbb{P}_{\beta, \ell, n}\left(x \leftrightarrow x+1 \mid \Omega_{\ell, n}^{\alpha}\right)-\mathbb{P}_{\beta, \ell, n}\left(x-1 \leftrightarrow x \mid \Omega_{\ell, n}^{\alpha}\right) \geq 1-2 \mathbb{P}_{\beta, \ell, n}\left(E_{x}^{\circlearrowleft} \mid \Omega_{\ell, n}^{\alpha}\right) .
$$

Proof Let $\omega \in \Omega_{\ell, n}^{\alpha}$. If $\omega \notin E_{x}^{\circlearrowleft}$, then $(x, 0)$ belongs to the exterior of all long loops and we have $x \leftrightarrow x+1$ and $x-1 \leftrightarrow x$. Then

$$
\begin{aligned}
& \mathbb{P}_{\beta, \ell, n}\left(x \leftrightarrow x+1 \mid \Omega_{\ell, n}^{\alpha}\right) \geq 1-\mathbb{P}_{\beta, \ell, n}\left(E_{x}^{\circlearrowleft} \mid \Omega_{\ell, n}^{\alpha}\right), \\
& \mathbb{P}_{\beta, \ell, n}\left(x-1 \leftrightarrow x \mid \Omega_{\ell, n}^{\alpha}\right) \geq 1-\mathbb{P}_{\beta, \ell, n}\left(E_{x}^{\circlearrowleft} \mid \Omega_{\ell, n}^{\alpha}\right) .
\end{aligned}
$$

The claim of the proposition follows immediately.

There remains to show that $\mathbb{P}_{\beta, \ell, n}\left(E_{x}^{\circlearrowleft} \mid \Omega_{\ell, n}^{\alpha}\right)$ is less than $\frac{1}{2}$, uniformly in $\beta, \ell, n$. 


\subsection{Blocks and domains}

We define a "block" to be a set $\{x, x+1\} \times I$, where $\{x, x+1\} \in E_{\ell}^{1}$ and $I$ is a proper interval in $T_{\beta, n}$. A "domain" $D$ is a finite collection of disjoint blocks.

Let $\Omega_{D}$ denote the set of configurations of double bars on $D$; that is, double bars within blocks of $D$, or between blocks involving nearest neighbors at equal times. We let $Z(D)$ denote the partition function on $D$, namely

$$
Z(D)=\sum_{\omega \in \Omega_{D}}\left(\frac{1}{n}\right)^{|\omega|}(2 S+1)^{\mathcal{L}_{D}(\omega)-|\omega|} .
$$

Loops are defined as before, with the understanding that bars are present at the ends of each block of $D$ and $\mathcal{L}_{D}(\omega)$ is the number of loops. Let $\mathbb{P}_{D}$ denote the probability measure on configurations on $D$. Let

$$
D_{\alpha}=\underset{\{x, x+1\} \in E_{\ell}^{1}}{\cup}\{x, x+1\} \times[\alpha+1, \alpha],
$$

where $[\alpha+1, \alpha]$ is the interval in $T_{\beta, n}$ that contains 0 .

Lemma 3.4 We have

$$
\mathbb{P}_{\beta, \ell, n}\left(E_{x}^{\circlearrowleft} \mid \Omega_{\ell, n}^{\alpha}\right)=\mathbb{P}_{D_{\alpha}}\left(E_{x}^{\circlearrowleft}\right) .
$$

Proof In the left side, the sum over $\omega \in \Omega_{\ell, n}^{\alpha}$ can be done by summing over $\omega \in \Omega_{D_{\alpha}}$, and by summing over configurations $\omega^{\prime}$ of double bars on $E_{\ell}^{1} \times[\alpha, \alpha+1]$. We have

$$
\begin{aligned}
& \sum_{\omega \in E_{x}^{\circlearrowleft} \cap \Omega_{\ell, n}^{\alpha}}\left(\frac{1}{n}\right)^{|\omega|}(2 S+1)^{\mathcal{L}(\omega)-|\omega|} \\
= & \sum_{\omega \in \Omega_{D_{\alpha}}} \sum_{\omega^{\prime}} 1_{E_{x}^{\circlearrowleft}}\left(\omega \cup \omega^{\prime}\right)\left(\frac{1}{n}\right)^{\left|\omega \cup \omega^{\prime}\right|}(2 S+1)^{\mathcal{L}\left(\omega \cup \omega^{\prime}\right)-\left|\omega \cup \omega^{\prime}\right|} .
\end{aligned}
$$

We have $\left|\omega \cup \omega^{\prime}\right|=|\omega|+\left|\omega^{\prime}\right|$ and

$$
\mathcal{L}\left(\omega \cup \omega^{\prime}\right)=\mathcal{L}_{D_{\alpha}}(\omega)+\left|\omega^{\prime}\right|-\ell .
$$

Finally, observe that the event $E_{x}^{\circlearrowleft}$ depends on $\omega$ only; the contribution of $\omega^{\prime}$ can be factored out and the lemma follows.

\section{Contour representation}

We now introduce a contour representation suitable for executing a Peierls argument. Developing a Peierls argument for our model is not entirely straightforward. In particular, since the cost of large contours is entropic, we need to estimate the sparsity of loops instead of relying on an energy estimate which is usually quite immediate. 

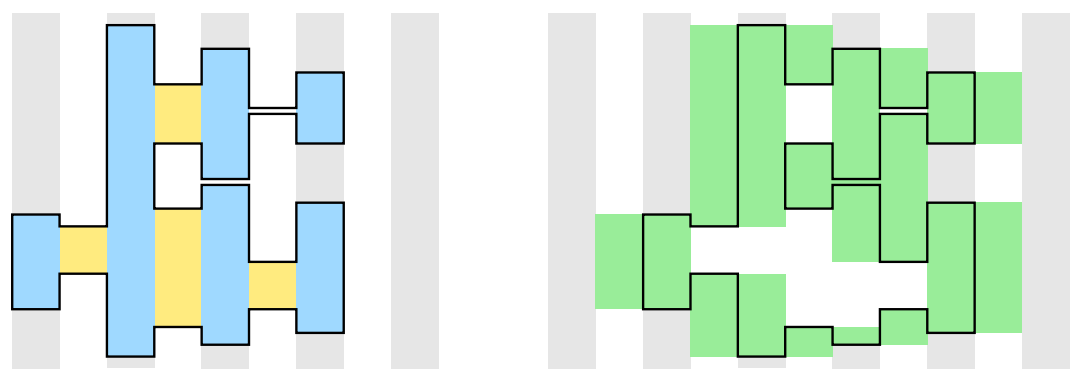

Fig. 4 (Color online) Left a contour $\gamma$ with its 1-interior int $1 \gamma$ (blue) and its 2-interior int $2 \gamma$ (yellow). Right the same contour with its support supp $\gamma$ (green)

We found it necessary to condition on arbitrary configurations of external contours not involving a given point $(x, 0)$. This allows to apply a simpler Peierls argument with a single contour, but in domains of arbitrary shape. We begin with a number of definitions.

An edge in space time is of the form $(\{x, x+1\}, t), x \in[-\ell, \ell-1] \subset \mathbb{Z}, t \in$ $[-\beta, \beta] \subset \mathbb{R}$. In figures it is more convenient to replace $\{x, x+1\}$ by $[x, x+1] \subset \mathbb{R}$. In the same way, in figures we will depict a block as a rectangular region in $\mathbb{R}^{2}$ of the form $[x, x+1] \times[a, b]$.

We now call contour a long loop; a contour $\gamma$ is characterized by

- its number of double bars, $|\gamma|$;

- its 1 -interior int $_{1} \gamma$, that is, the set of edges $\{x, x+1\} \times t$ inside the enclosed area, with $\{x, x+1\} \in E_{\ell}^{1}$;

- its 2-interior int $2 \gamma$, that is, the set of edges $\{x, x+1\} \times t$ inside the enclosed area, with $\{x, x+1\} \in E_{\ell}^{2}$;

- the length $L(\gamma)$ such that $\mid$ int $_{1} \gamma|-|$ int $_{2} \gamma \mid=\frac{1}{2} n L(\gamma)$, where $|\cdot|$ means cardinality. $L(\gamma)$ is almost equal to the lengths of vertical legs of the loop (it is equal up to $\left.\frac{|\gamma|}{n}\right)$, because each stretch of the boundary of $i_{1} \gamma$ belongs either to the perimeter or to the vertical boundary of int $_{2} \gamma$;

- its support $\operatorname{supp} \gamma$, the set of edges of $E_{\ell}$ with at least one endpoint on the loop;

- its exterior ext $\gamma$, which is equal to the union of blocks outside the enclosed area;

A contour with its interiors and its support is displayed in Fig. 4. Notice that the event $E_{x}^{\circlearrowleft}$ is equivalent to

$$
E_{x}^{\circlearrowleft}=\left\{\omega \in \Omega_{\ell, n}^{\alpha}: \exists \text { contour } \gamma \text { such that }(x, 0) \in \text { int }_{1} \gamma\right\} .
$$

Given $\omega \in \Omega_{\ell, n}^{\alpha}$, we call "external contour" a loop such that

- it involves at least a jump $\{x, x+1\} \in E_{\ell}^{2}$;

- it is not surrounded by another loop.

This definition is illustrated in Fig. 5. We identify an external contour $\gamma$ with the set of double bars that it involves; that is, $\gamma$ denotes both a closed trajectory and an element of $\Omega_{D_{\alpha}}$. 


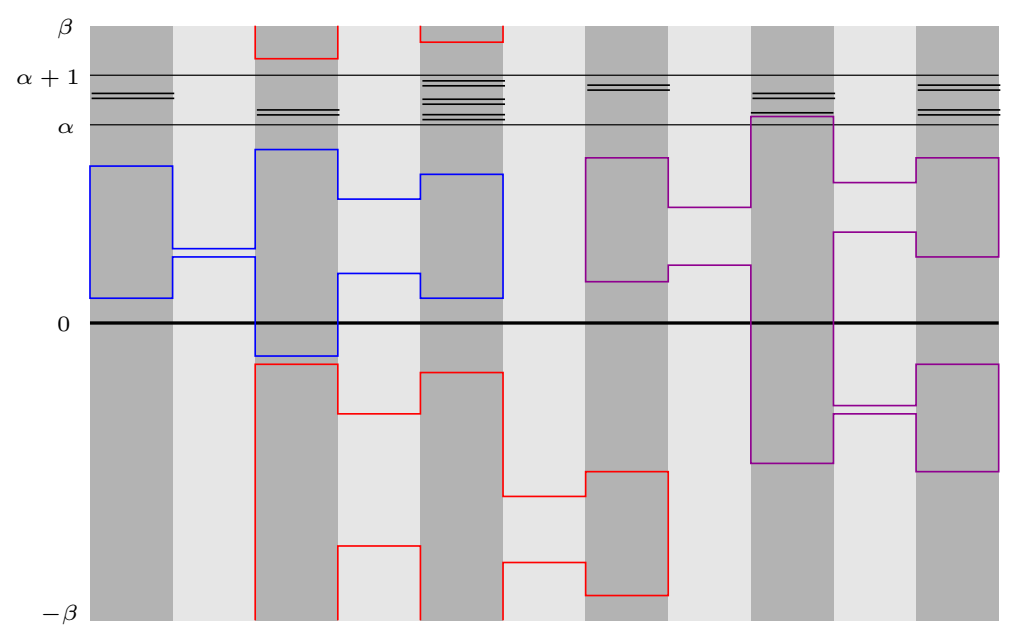

Fig. 5 A configuration of $\Omega_{\ell, n}^{\alpha}$ with three external contours

A difficulty with our Peierls argument is to control the change in the number of loops when erasing a contour. We find it convenient to condition on the configuration of external contours away from $(x, 0)$; a similar idea was used in [14]. This gives a domain with complicated shape, but this is not a problem. The background configuration of loops is now simpler and this is useful. Given $\omega \in \Omega_{D_{\alpha}}$, consider the set $\Gamma$ of external contours that do not surround $(x, 0)$. Let ext $\Gamma=D_{\alpha} \backslash \operatorname{int}_{1} \Gamma$, and let $X(\omega) \subset$ ext $\Gamma$ denote the connected component that contains $(x, 0)$. That is, the domain $X(\omega)$ is a subset of $D_{\alpha}$, and the graph whose vertices are the blocks, and with edges between blocks that can be connected by a double bar, is connected. This is illustrated in Fig. 6. Finally, let $\widetilde{\Omega}_{X} \subset \Omega_{X}$ denote the configurations without contours, or with just one external contour that surrounds $(x, 0)$; and let $\widetilde{\mathbb{P}}_{X}(\cdot)=\mathbb{P}_{X}\left(\cdot \mid \widetilde{\Omega}_{X}\right)$ denote the conditional probability.

Lemma 4.1 There exists $p_{X} \geq 0$ such that $\sum_{X} p_{X}=1$ and

$$
\mathbb{P}_{D_{\alpha}}\left(E_{x}^{\circlearrowleft}\right)=\sum_{X} p_{X} \widetilde{\mathbb{P}}_{X}\left(E_{x}^{\circlearrowleft}\right)
$$

Proof We have

$$
\mathbb{P}_{D_{\alpha}}\left(E_{x}^{\circlearrowleft}\right)=\frac{1}{Z\left(D_{\alpha}\right)} \sum_{X} \sum_{\substack{\omega: X(\omega)=X \\ \omega \mid X=\emptyset}} \sum_{\omega^{\prime} \in \widetilde{\Omega}_{X} \cap E_{x}^{\circlearrowleft}}\left(\frac{1}{n}\right)^{\left|\omega \cup \omega^{\prime}\right|}(2 S+1)^{\mathcal{L}_{D_{\alpha}}\left(\omega \cup \omega^{\prime}\right)-\left|\omega \cup \omega^{\prime}\right|} .
$$

Let $b(X)$ the number of blocks of $X$; the number of loops of $\omega \cup \omega^{\prime}$ satisfies the important relation

$$
\mathcal{L}_{D_{\alpha}}\left(\omega \cup \omega^{\prime}\right)=\mathcal{L}_{D_{\alpha}}(\omega)+\mathcal{L}_{X}\left(\omega^{\prime}\right)-b(X) .
$$




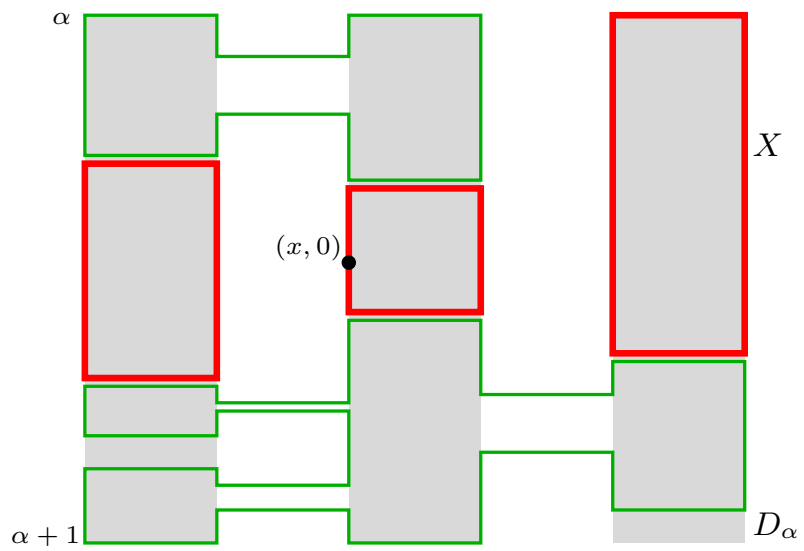

Fig. 6 Set $X$ around $(x, 0)$

Indeed, this holds for $\omega^{\prime}=\emptyset$ since $\mathcal{L}_{X}(\emptyset)=b(X)$; adding a double bar to $\omega^{\prime}$ results in the same change in $\mathcal{L}_{D_{\alpha}}\left(\omega \cup \omega^{\prime}\right)$ and $\mathcal{L}_{X}\left(\omega^{\prime}\right)$.

Let $\widetilde{Z}(X)$ be the partition function on $\widetilde{\Omega}_{X}$; we have

$$
\begin{aligned}
& \mathbb{P}_{D_{\alpha}}\left(E_{x}^{\circlearrowleft}\right)=\sum_{X} \frac{1}{Z\left(D_{\alpha}\right)} \sum_{\substack{\omega: X(\omega)=\left.X \\
\omega\right|_{X}=\emptyset}}\left(\frac{1}{n}\right)^{|\omega|}(2 S+1)^{\mathcal{L}_{D_{\alpha}}(\omega)-|\omega|-b(X)} \tilde{Z}(X) \\
& \frac{1}{\widetilde{Z}(X)} \sum_{\omega^{\prime} \in \widetilde{\Omega}_{X} \cap E_{X}^{\circlearrowleft}}\left(\frac{1}{n}\right)^{\left|\omega^{\prime}\right|}(2 S+1)^{\mathcal{L}_{X}\left(\omega^{\prime}\right)-\left|\omega^{\prime}\right|} .
\end{aligned}
$$

For given $X$, the first line of the right side gives $p_{X}$; the second line is $\widetilde{\mathbb{P}}_{X}\left(E_{x}^{\circlearrowleft}\right)$ and we obtain the lemma.

In view of Proposition 3.3, to prove Theorem 2.1 we need to show that, if $S \geq 40$, we have $\widetilde{\mathbb{P}}_{X}\left(E_{x}^{\circlearrowleft}\right)<\frac{1}{2}$ for all $X$. This will be achieved in Proposition 4.4. In preparation for that estimate, we need two lemmas.

If $\widetilde{\omega} \in \Omega_{X}$ is a configuration that contains the contour $\gamma$, then it is the disjoint union

$$
\widetilde{\omega}=\gamma \cup \omega \cup \omega^{\prime}
$$

where $\omega \in \Omega_{\text {ext } \gamma}$ and $\omega^{\prime} \in \Omega_{\text {int }_{2} \gamma}$. Let rint $_{2} \gamma$ denote the shift of int $2 \gamma$ by one unit to the right; if $\omega^{\prime} \in \Omega_{\text {int }_{2} \gamma}$, we also let $\mathrm{r} \omega^{\prime} \in \Omega_{\text {int }_{2} \gamma}$ denote the right shift of $\omega^{\prime}$ by one unit. The next lemma is stated and proved for any configurations of $\Omega_{X}$, but we only need it for configurations of $\widetilde{\Omega}_{X}$.

Lemma 4.2 Let $\gamma$ be an external contour, $\omega \in \Omega_{\mathrm{ext} \gamma}$, and $\omega^{\prime} \in \Omega_{\mathrm{int}_{2} \gamma}$. Then the configuration $\gamma \cup \omega \cup \omega^{\prime} \in \Omega_{X}$ satisfies

$$
\mathcal{L}_{X}\left(\gamma \cup \omega \cup \omega^{\prime}\right) \leq \mathcal{L}_{X}\left(\omega \cup \mathrm{r} \omega^{\prime}\right)+\frac{1}{2}|\gamma|+1
$$




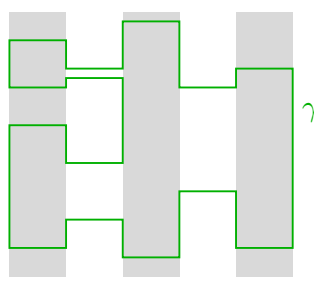

(a)

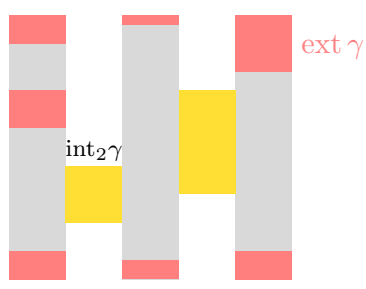

(b)

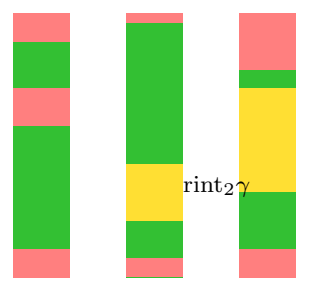

(c)

Fig. 7 a A contour $\gamma$; b the blocks of ext $\gamma$ (red) and int $2 \gamma$ (yellow); $\mathbf{c}$ the blocks of ext $\gamma$ (red) and rint $2 \gamma$ (yellow), and the green blocks (color figure online)

Proof See Fig. 7; we have

$$
\mathcal{L}_{X}\left(\gamma \cup \omega \cup \omega^{\prime}\right)=1+\mathcal{L}_{\text {ext } \gamma \cup \operatorname{rint}}{ }_{2} \gamma\left(\omega \cup \mathrm{r} \omega^{\prime}\right)
$$

We call "green blocks" the connected components of $\operatorname{int}_{1} \gamma \backslash \operatorname{rint}_{2} \gamma$. We add these blocks one by one to the domain; at each step, the number of loops may decrease by one, so that $\mathcal{L}_{\text {ext } \gamma \cup \operatorname{rint}_{2} \gamma}\left(\omega \cup \mathrm{r} \omega^{\prime}\right) \leq \mathcal{L}_{X}\left(\omega \cup \mathrm{r} \omega^{\prime}\right)+$ \#green blocks. The number of green blocks is less than $\frac{1}{2}|\gamma|$.

The following lemma is motivated by a Peierls argument. The probability that $(x, 0)$ is surrounded by a contour can be bounded by a sum over these contours with exponentially small weights.

\section{Lemma 4.3}

$$
\widetilde{\mathbb{P}}_{X}\left(E_{x}^{\circlearrowleft}\right) \leq(2 S+1) \sum_{\gamma \in \widetilde{\Omega}_{X} \cap E_{x}^{\circlearrowleft}}\left(\frac{1}{n}\right)^{|\gamma|}(2 S+1)^{-\frac{1}{2}|\gamma|}\left(1+\frac{1}{n}\right)^{-\frac{1}{2} n L(\gamma)} .
$$

Proof We have

$$
\begin{aligned}
& \sum_{\omega^{\prime} \in \widetilde{\Omega}_{X} \cap E_{x}^{\circlearrowleft}}\left(\frac{1}{n}\right)^{\left|\omega^{\prime}\right|}(2 S+1)^{\mathcal{L}_{X}\left(\omega^{\prime}\right)-\left|\omega^{\prime}\right|} \\
& =\sum_{\gamma \in \widetilde{\Omega}_{X} \cap E_{x}^{\circlearrowleft}} \sum_{\omega \in \Omega_{\mathrm{ext} \gamma}} \sum_{\omega^{\prime} \in \Omega_{\mathrm{int}} \gamma}\left(\frac{1}{n}\right)^{\left|\gamma \cup \omega \cup \omega^{\prime}\right|}(2 S+1)^{\mathcal{L}_{X}\left(\gamma \cup \omega \cup \omega^{\prime}\right)-\left|\gamma \cup \omega \cup \omega^{\prime}\right|} \\
& \leq(2 S+1) \sum_{\gamma \in \widetilde{\Omega}_{X} \cap E_{x}^{\circlearrowleft}}\left(\frac{1}{n}\right)^{|\gamma|}(2 S+1)^{-\frac{1}{2}|\gamma|} \\
& \quad \times \sum_{\omega \in \Omega_{\mathrm{ext}} \gamma} \sum_{\omega^{\prime} \in \Omega_{\mathrm{int}_{2} \gamma}}\left(\frac{1}{n}\right)^{\left|\omega \cup \omega^{\prime}\right|}(2 S+1)^{\mathcal{L}_{X}\left(\omega \cup \omega^{\prime}\right)-\left|\omega \cup \omega^{\prime}\right|} .
\end{aligned}
$$


The inequality follows from Lemma 4.2. We now obtain a lower bound for $\widetilde{Z}(X)$. Let $A(\gamma)=$ int $_{1} \gamma \backslash$ rint $_{2} \gamma$; since $|A(\gamma)|=\frac{1}{2} n L(\gamma)$, we have

$$
\begin{aligned}
\widetilde{Z}(X) & =\sum_{\omega \in \widetilde{\Omega}_{X}}\left(\frac{1}{n}\right)^{|\omega|}(2 S+1)^{\mathcal{L}_{X}(\omega)-|\omega|} \\
& \geq \sum_{\omega \in \Omega_{\text {ext }}} \sum_{\omega^{\prime} \in \Omega_{\text {rint }_{2} \gamma}} \sum_{\omega^{\prime \prime} \in \Omega_{A}(\gamma)}\left(\frac{1}{n}\right)^{\left|\omega \cup \omega^{\prime} \cup \omega^{\prime \prime}\right|}(2 S+1)^{\mathcal{L}_{X}\left(\omega \cup \omega^{\prime} \cup \omega^{\prime \prime}\right)-\left|\omega \cup \omega^{\prime} \cup \omega^{\prime \prime}\right|} .
\end{aligned}
$$

We restrict the sum over $\omega^{\prime \prime}$ so that double bars are within single blocks of $A(\gamma)$, that is, $\omega^{\prime \prime} \subset A(\gamma)$. Then $\mathcal{L}_{X}\left(\omega \cup \omega^{\prime} \cup \omega^{\prime \prime}\right)=\mathcal{L}_{X}\left(\omega \cup \omega^{\prime}\right)+\left|\omega^{\prime \prime}\right|$; this holds true because $\omega$ contains only simple loops and no contours. The sum over $\omega^{\prime \prime}$ gives $\left(1+\frac{1}{n}\right)^{|A(\gamma)|}$, and we obtain the estimate claimed in the statement.

\section{Proposition 4.4 We have}

$$
\lim _{n \rightarrow \infty} \mathbb{P}_{\beta, \ell, n}\left(E_{x}^{\circlearrowleft} \mid \Omega_{\ell, n}^{\alpha}\right) \leq \frac{64}{(2 S+1)^{3 / 2}}+\frac{128}{(2 S+1)^{2}}+\frac{1}{12} \sum_{k \geq 7} \frac{(k+1) 4^{k}}{(2 S+1)^{\frac{1}{2} k-1}}
$$

Proof Starting with the upper bound in Lemma 4.3, we explicitly perform the sum over the external contour $\gamma$ that surrounds $(x, 0)$. Let us assign a direction to the loops, namely they go "up" on top of the sites $\{-\ell+1,-\ell+3, \ldots, \ell-1\}$ and "down" on top of the sites $\{-\ell+2,-\ell+4, \ldots, \ell\}$. Let $y$ be the leftmost site where the loop crosses the $t=0$ line and moves up. We can choose $\gamma$ by summing over numbers $t_{1}, \ldots, t_{k}$ of time intervals $\frac{1}{n}$ between jumps (not counting the ends of the blocks of $X$ ) and by choosing the $k-2$ directions of the jumps (the first and last directions are forced). Then

$$
\begin{aligned}
& \sum_{\gamma \in \widetilde{\Omega}_{X} \cap E_{x}^{\circlearrowleft}}\left(\frac{1}{n}\right)^{|\gamma|}(2 S+1)^{-\frac{1}{2}|\gamma|}\left(1+\frac{1}{n}\right)^{-\frac{1}{2} n L(\gamma)} \\
& \quad \leq \sum_{y \leq x} \sum_{k \geq|x-y|} 2^{k-2}\left(\frac{1}{n}\right)^{k}(2 S+1)^{-\frac{1}{2} k} \sum_{t_{1}, \ldots, t_{k} \geq 1} \prod_{i=1}^{k}\left(1+\frac{1}{n}\right)^{-\frac{1}{2} t_{i}} .
\end{aligned}
$$

Since $y \in\{-\ell+1,-\ell+3, \ldots, \ell-1\}$, there are less than $\frac{1}{3}(k+1)$ possibilities. Each sum over $t_{i}$ gives $\left[1-\left(1+\frac{1}{n}\right)^{-1 / 2}\right]^{-1}$. It gets multiplied by $\frac{1}{n}$, which gives 2 in the limit $n \rightarrow \infty$. The contribution of contours with $k$ double bars is then less than $\frac{1}{12}(k+1) 4^{k}(2 S+1)^{1-\frac{1}{2} k}$. The smallest contours have $k=5$; they actually have limited entropy, so the contribution of contours with $k=5$ and $k=6$ is less than $2^{k+1}(2 S+1)^{1-\frac{1}{2} k}$. This gives the bound of the lemma. 
Proof of Theorem 2.1 From Proposition 3.1, Lemma 3.2, and Proposition 3.3, we have for $x \in\{-\ell+3, \ldots, \ell-1\}$ that

$$
\left\langle P_{x, x+1}^{(0)}\right\rangle_{\infty, \ell}-\left\langle P_{x-1, x}^{(0)}\right\rangle_{\infty, \ell} \geq\left(1-\frac{1}{(2 S+1)^{2}}\right) \inf _{\alpha} \lim _{\beta \rightarrow \infty} \lim _{n \rightarrow \infty}\left[1-2 \mathbb{P}_{\beta, \ell, n}\left(E_{x}^{\circlearrowleft} \mid \Omega_{\ell, n}^{\alpha}\right)\right] .
$$

The result follows from Proposition 4.4. Indeed, the upper bound is finite when $S>\frac{15}{2}$ and it is decreasing in $S$; it is equal to $\frac{1}{2}$ at $S=39.2$.

Proof of Theorem 2.2 In a similar fashion as Proposition 3.1, we can show that

$$
\left.\left\langle S_{x}^{i} S_{y}^{j}\right\rangle_{\beta, \ell}\right\rangle=\frac{1}{3} S(S+1) \delta_{i, j}(-1)^{x-y} \mathbb{P}_{\beta, \ell, n}(x \leftrightarrow y)
$$

This formula was explicitly derived in [4] and [25]. For $x$ and $y$ to be connected, if $|x-y| \geq 2$, there must exists a contour $\gamma$ that surrounds $x$ and such that $|\gamma|$ is greater than $|x-y|$. By Lemmas 3.4, 4.1, and 4.3, we obtain

$$
\begin{aligned}
& \mathrm{e}^{|x-y| / \eta} \mathbb{P}_{\beta, \ell, n}\left(x \leftrightarrow y \mid \Omega_{\ell, n}^{\alpha}\right) \\
& \leq(2 S+1) \sum_{\gamma \in \tilde{\Omega}_{X} \cap E_{x}^{\circlearrowleft}}\left(\mathrm{e}^{1 / \eta} \frac{1}{n}\right)^{|\gamma|}(2 S+1)^{-\frac{1}{2}|\gamma|}\left(1+\frac{1}{n}\right)^{-\frac{1}{2} n L(\gamma)} .
\end{aligned}
$$

We can adapt the proof of Proposition 4.4. The only difference is a factor $\mathrm{e}^{k / \eta}$, so we obtain

$$
\lim _{n \rightarrow \infty} \mathrm{e}^{|x-y| / \eta} \mathbb{P}_{\beta, \ell, n}\left(x \leftrightarrow y \mid \Omega_{\ell, n}^{\alpha}\right) \leq \frac{1}{12} \sum_{k \geq|x-y|} \frac{(k+1)\left(4 \mathrm{e}^{1 / \eta}\right)^{k}}{(2 S+1)^{\frac{1}{2} k-1}} .
$$

The series converges when $S>\frac{15}{2}$ and $\eta$ is large enough. The theorem follows from Lemma 3.2.

\section{Discussion}

For the spin chain with Hamiltonian (1.1), we established the existence of dimerization when $S \geq 8$ : in the thermodynamic limit the model has at least two 2-periodic ground states in which the translation invariance is broken. This follows directly from Theorem 2.1. We do not expect that these models have other ground states. In particular, based on what has been shown for the antiferromagnetic $X X Z$ chain [10], it seems unlikely that domain-wall ground states exist for this model. We also proved that in the two ground states we constructed, the $S U(2)$-invariance remains unbroken.

As stated in Theorem 2.2, our proof of dimerization also implies exponential decay of correlations in the ground states. Following the arguments of Kennedy and Tasaki [15], exponential decay implies a spectral gap in this setting.

One may also ask about the stability of the dimerization under small translationinvariant perturbations of the interaction. Since the model is not frustration free and 
involves translation symmetry breaking, the result of Michalakis and Zwolak [20] does not apply but we expect that the random loop representation can be used as a starting point for studying perturbations of the model using traditional cluster expansion techniques as is done, e.g., in [15]. We have not pursued this possibility as it is beyond the scope of this work, but establishing stability under arbitrary, uniformly bounded, and sufficiently small perturbation of the interactions would certainly be un important contribution to understanding the phase diagram of quantum spin chains [2].

Acknowledgements The research reported in this article was completed during the workshop Many-Body Quantum Systems and Effective Theories at the Mathematisches Forschungsinstitut Oberwolfach, September 11-17, 2016. We thank the organizers and the institute for the great hospitality and the stimulating program. One referee made very useful comments. The work of BN was supported in part by the National Science Foundation under Grant DMS-1515850.

Open Access This article is distributed under the terms of the Creative Commons Attribution 4.0 International License (http://creativecommons.org/licenses/by/4.0/), which permits unrestricted use, distribution, and reproduction in any medium, provided you give appropriate credit to the original author(s) and the source, provide a link to the Creative Commons license, and indicate if changes were made.

\section{References}

1. Affleck, I.: Exact results on the dimerisation transition in $s u(n)$ antiferromagnetic chains. J. Phys. Condens. Matter 2, 405-415 (1990)

2. Affleck, I., Haldane, F.D.M.: Critical theory of quantum spin chains. Phys. Rev. B 36, 5291-5300 (1987)

3. Affleck, I., Lieb, E.H.: A proof of part of Haldane's conjecture on spin chains. Lett. Math. Phys. 12, 57-69 (1986)

4. Aizenman, M., Nachtergaele, B.: Geometric aspects of quantum spin states. Comm. Math. Phys. 164, 17-63 (1994)

5. Barber, M.N., Batchelor, M.T.: Spectrum of the biquadratic spin-1 antiferromagnetic chain. Phys. Rev. B 40, 4621-4626 (1989)

6. Baxter, R.J.: Potts model at the critical temperature. J. Phys. C 6, L445-L448 (1973)

7. Björnberg, J.E., Ueltschi, D.: Decay of transverse correlations in quantum Heisenberg models. J. Math. Phys. 56, 043303 (2015)

8. Crawford, N., Ng, S., Starr, S.: Emptiness formation probability. Commun. Math. Phys. 345, 881-922 (2016)

9. Cross, M.C., Fisher, D.S.: A new theory of the spin-Peierls transition with special relevance to the experiments on TTFCuBDT. Phys. Rev. B 19, 402-419 (1979)

10. Datta, N., Kennedy, T.: Expansions for one quasiparticle states in spin $1 / 2$ systems. J. Stat. Phys. 108, 373-399 (2002)

11. Dyson, F.J., Lieb, E.H., Simon, B.: Phase transitions in quantum spin systems with isotropic and nonisotropic interactions. J. Stat. Phys. 18, 335-383 (1978)

12. Frank, R.L., Lieb, E.H.: Possible lattice distortions in the Hubbard model for graphene. Phys. Rev. Lett. 107, 066801 (2011)

13. Giuliani, A., Lieb, E.H.: Columnar phase in quantum dimer models. J. Phys. A Math. Theor. 48, 235203 (2015)

14. Giuliani, A., Seiringer, R.: Periodic striped ground states in Ising models with competing interactions. Commun. Math. Phys. 347, 983-1007 (2016)

15. Kennedy, T., Tasaki, H.: Hidden symmetry breaking and the Haldane phase in $s=1$ quantum spin chains. Commun. Math. Phys. 147, 431-484 (1992)

16. Klümper, A.: The spectra of $q$-state vertex models and related antiferromagnetic quantum spin chains the spectra of q-state vertex models and related antiferromagnetic quantum spin chains. J. Phys. A Math. Gen. 23, 809-823 (1990) 
17. Korepin, V.E., Bogoliubov, N.M., Izergin, A.G.: Quantum inverse scattering method and correlation functions. Cambridge University Press, Cambridge (1993)

18. Kotecký, R., Shlosman, S.B.: First-order phase transitions in large entropy lattice models. Commun. Math. Phys. 83, 493-515 (1982)

19. Lees, B.: Existence of Néel order in the $S=1$ bilinear-biquadratic Heisenberg model via random loops. Commun. Math. Phys. 347, 83-101 (2016)

20. Michalakis, S., Zwolak, J.P.: Stability of frustration-free Hamiltonians. Commun. Math. Phys. 322, 277-302 (2013)

21. Nachtergaele, B.: Quasi-state decompositions for quantum spin systems, Probability theory and mathematical statistics. In: Grigelionis, B. et al. (eds.) Proceedings of the 6th Vilnius Conference, VSP/TEV, Utrecht-Tokyo-Vilnius, pp. 565-590 (1994)

22. Nepomechie, R.I., Pimenta, R.A.: Universal Bethe ansatz solution for the temperley-lieb spin chain. Nucl. Phys. B 910, 910-928 (2016)

23. Ralko, A., Poilblanc, D., Moessner, R.: Generic mixed columnar-plaquette phases in Rokhsar-Kivelson models. Phys. Rev. Lett. 100, 037201 (2008)

24. Sirker, J., Klümper, A., Hamacher, K.: Ground-state properties of two-dimensional dimerized Heisenberg models. Phys. Rev. B 65, 134409 (2002)

25. Ueltschi, D.: Random loop representations for quantum spin systems. J. Math. Phys. 54, 083301 (2013) 